\title{
TRATAMIENTO CON TERAPIA FOTODINÁMICA EN LAS ESTRÍAS ANGIOIDES
}

\section{TREATMENT OF ANGIOID STREAKS WITH PHOTHODYNAMIC THERAPY}

\author{
GONZÁLEZ-BLANCO MJ ${ }^{1}$, BLANCO-RIVERA C ${ }^{2}$, CAMPOS-GARCÍA S ${ }^{2}$
}

\section{RESUMEN}

Caso clínico: Paciente con estrías angioides bilaterales complicadas con degeneración cicatricial en el ojo izquierdo y posterior aparición de una membrana neovascular subfoveal en el ojo derecho. Se plantea tratamiento con terapia fotodinámica y la paciente responde favorablemente con recuperación de la agudeza visual y regresión angiográfica de la lesión.

Discusión: Las membranas neovasculares son la complicación más grave de las estrías angioides. Hoy en día uno de los posibles tratamientos de las membranas neovasculares subfoveales es la terapia fotodinámica, siendo ésta, también, un tratamiento eficaz en algunas membranas neovasculares subfoveales secundarias a estrías angioides.

Palabras clave: Estrías angioides, membrana neovascular subfoveal, terapia fotodinámica, angiografía fluoresceínica, oftalmoscopia.

\begin{abstract}
Clinical case: A patient had bilateral angioid streaks complicated by cicatricial degeneration in the left eye and subfoveal choroidal neovascularization in the right eye. Photodynamic therapy resulted in a favorable response with normalization of the visual acuity and angiographic resolution of the lesions.

Discussion: The most serious complication of angioid streaks is choroidal neovascularization. Today photodynamic therapy is an effective treatment of subfoveal choroidal neovascularization. It also appears useful in the treatment of choroidal neovascularization in angioid streaks (Arch Soc Esp Oftalmol 2007; 82: 719-722).
\end{abstract}

Key words: Angioid streaks, subfoveal choroidal neovascularization, photodynamic therapy, fluorescein angiography, ophthalmoscopic.

\section{INTRODUCCIÓN}

Doyne describió en 1889 las estrías angioides como líneas oscuras e irregulares a nivel peripapi- lar. Posteriormente se sospechó que la lesión se localizaba a nivel de la membrana de Bruch. Actualmente se asocian a una degeneración primaria de fibras elásticas y ruptura de la membrana de

\footnotetext{
Recibido: 31/5/06. Aceptado: 17/8/07.

Complejo Hospitalario Universitario de Vigo (CHUVI). Servicio de Oftalmología. Vigo (Pontevedra). España.

1 Licenciado en Medicina.

2 Doctor en Medicina.

Comunicación presentada en el LXXXII Congreso de la S.E.O. (La Coruña 2006).
}

Correspondencia:

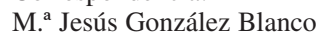

Hospital Xeral-Cíes, Servicio de Oftalmología

C/. Pizarro, 22

36204 Vigo (Pontevedra)

España

E-mail: chus_gb@yahoo.es 
Bruch. Etiológicamente pueden ser idiopáticas (25$50 \%$ ) o estar asociadas a múltiples enfermedades sistémicas, entre las que destaca por su frecuencia el Pseudoxantoma elástico (1). Clínicamente suelen pasar desapercibidas en estadios iniciales, pero en estadios avanzados cursan con pérdida de agudeza visual y metamorfopsias por afectación foveal. Suelen ser bilaterales y asimétricas e ir acompañadas de alteraciones como «piel de naranja», «manchas salmón», drusas papilares, agrupación periférica reticular, etc. Su evolución es muy variable, pudiendo permanecer estables o complicarse, siendo la complicación más frecuente las membranas neovasculares subretinianas (2).

La terapia fotodinámica (TFD) puede constituir uno de los tratamientos de elección en algunas membranas neovasculares subfoveales $(3,4)$ que, hasta antes de la aplicación de este tratamiento, marcaban de forma negativa el pronóstico de esta patología.

\section{CASO CLÍNICO}

Mujer de 43 años que acudió por pérdida progresiva de agudeza visual en el ojo izquierdo. Como antecedente destacaba el diagnóstico de estrías angioides cinco años antes. En la exploración oftalmológica se objetivó una agudeza visual lejana de unidad en el ojo derecho y de «contar dedos» a dos metros en el ojo izquierdo. La tensión ocular y la biomicroscopía estaban dentro de los parámetros normales. La oftalmoscopia bajo dilatación mostró, en ambos ojos, la existencia de lesiones lineales serpenteantes radiales al disco óptico que se extendían hacia la periferia. Además, en el ojo izquierdo, se apreciaba una lesión cicatricial de gran tamaño con áreas de movilización de pigmento y fibrosis que englobaba toda el área macular (fig. 1). Se realizó una angiografía fluoresceínica (AGF) con los siguientes resultados: en el ojo derecho se apreciaron unas líneas hipo e hiperfluorescentes con movilización de pigmento a nivel macular sin difusión de contraste. En el ojo izquierdo se observaron la mismas lesiones lineales acompañadas de un área hipo e hiperfluorescente a nivel macular sin difusión de contraste en tiempos tardíos (fig. 2) siendo el diagnóstico compatible con estrías angioides bilaterales y además, en el ojo izquierdo, complicadas con una membrana neovascular subretiniana no susceptible de tratamiento. Se aconsejó el seguimiento clínico periódico.

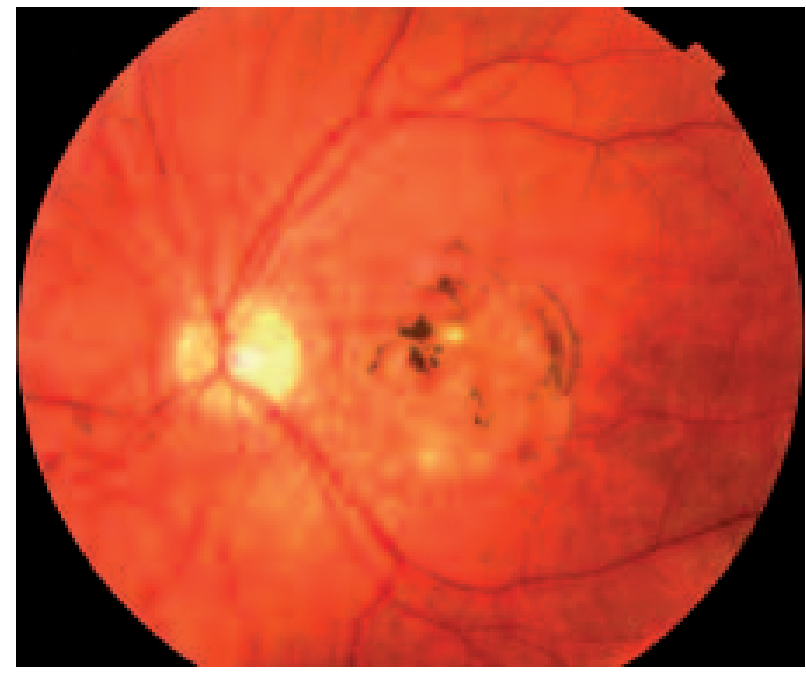

Fig. 1: Fondo de ojo izquierdo de la paciente. Estrías anaranjadas y lesión cicatricial macular.

Dos meses después la paciente acudió refiriendo empeoramiento de la agudeza visual, esta vez, en el ojo derecho. La exploración oftalmológica reveló una agudeza visual de unidad con dificultad en el ojo derecho y de «contar dedos» a dos metros en el ojo izquierdo. Funduscópicamente, en el ojo derecho, se apreció una pequeña hemorragia y movilización de pigmento en polo posterior con estrías anaranjadas, ya presentes en exploraciones previas (fig. 3). Se realizó una nueva AGF del ojo derecho que puso de manifiesto una hiperfluorescencia a

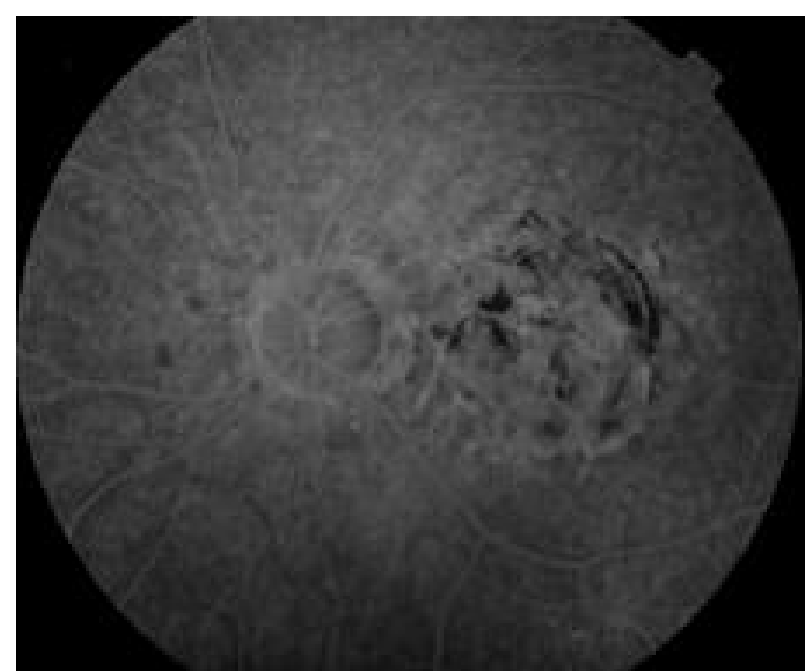

Fig. 2: Imagen angiográfica del ojo izquierdo. Estrías hipo e hiperfluorescentes y lesión cicatricial a nivel macular sin difusión de contraste. 


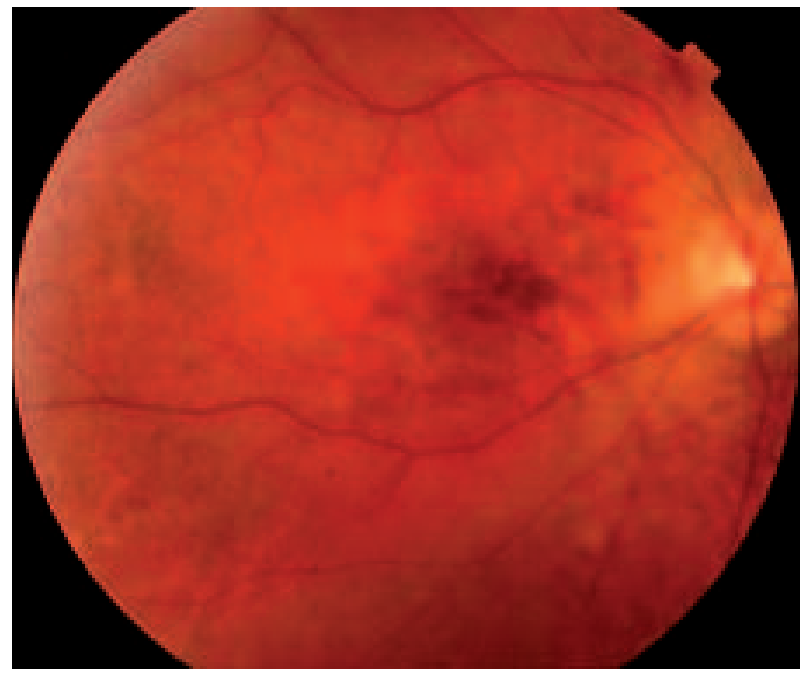

Fig. 3: Fondo de ojo derecho dos meses después de la primera consulta. Estrías anaranjadas radiales al disco óptico, hemorragia y movilización de pigmento a nivel macular.

nivel macular desde tiempos iniciales rodeada de un halo hipofluorescente con aumento de la fluorescencia hacia tiempos tardíos y mínima difusión, no existente en AGF previas, compatible con una membrana neovascular subfoveal (fig. 4). Se estableció, por tanto, un nuevo diagnóstico, estrías angioides complicadas con membrana neovascular subfoveal en ojo derecho. Se le planteó el tratamiento con TFD y tras la primera sesión se produjo la reabsorción parcial de la hemorragia con mantenimiento de la pigmentación central. Dichos hallazgos se confirmaron con la AGF que reveló una ligera difusión de contraste en tiempos tardíos (fig. 5). Por ello, a pesar de la recuperación visual, se decidió aplicar dos sesiones más de TFD. En la última revisión la agudeza visual se mantenía en la unidad y angiográficamente se observaba una lesión hipo e

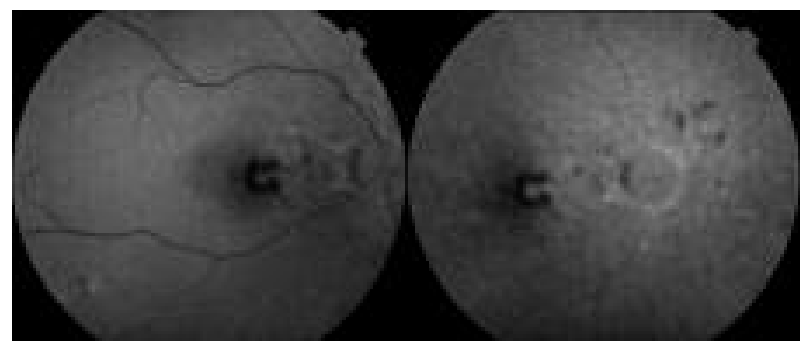

Fig. 4: Fase angiográfica precoz (derecha) y tardía (izquierda) de membrana neovascular subfoveal en el ojo derecho dos meses después de la primera consulta.

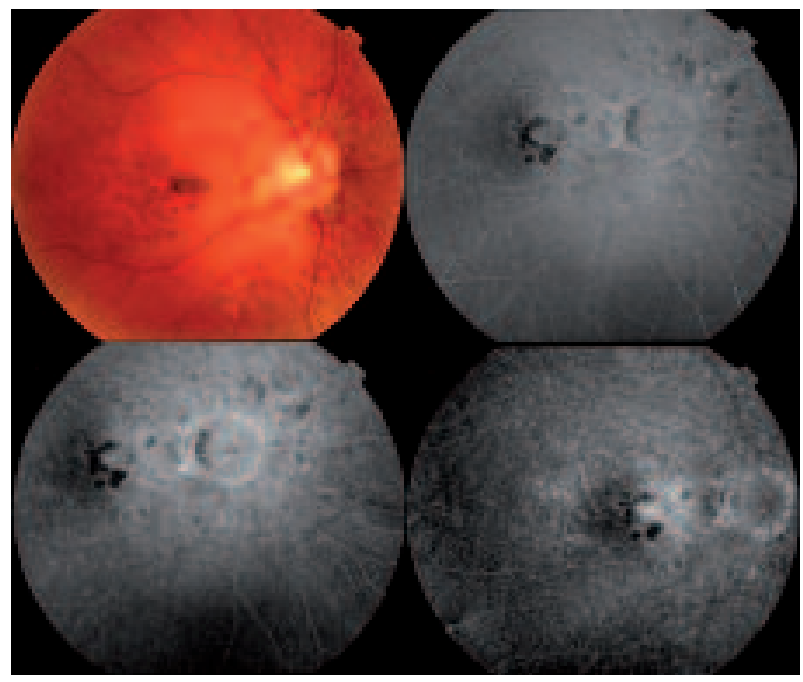

Fig. 5: Fondo de ojo derecho y secuencia angiográfica tras primera sesión de terapia fotodinámica.

hiperfluorescente compatible con una lesión cicatricial en el área macular. En tiempos angiográficos medios aparecía un aumento de la hiperfluorescencia que se mantenía en tiempos tardíos, pero sin difusión de contraste (fig. 6).

\section{DISCUSIÓN}

La principal y más grave complicación de las estrías angioides la constituyen las membranas neovasculares subretinianas, siendo, además, la princi-

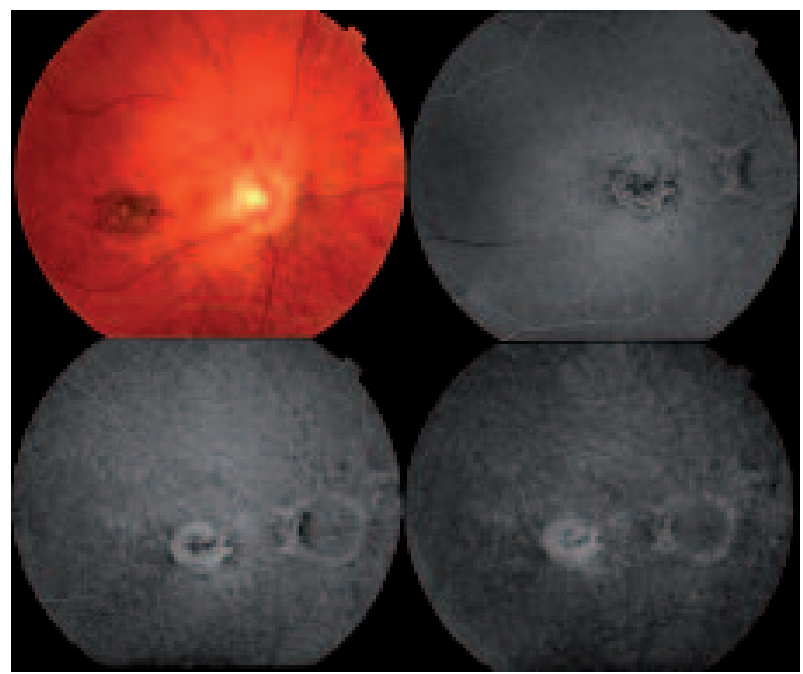

Fig. 6: Fondo de ojo derecho y secuencia angiográfica tras la última sesión de terapia fotodinámica. 
pal causa de ceguera en estos enfermos. Para establecer el diagnóstico de las estrías o de sus complicaciones resulta imprescindible el estudio oftalmoscópico y angiográfico (3).

En nuestro caso clínico, la AGF ha resultado imprescindible para establecer el diagnóstico final, realizar el tratamiento y objetivar la eficacia del mismo. Dada la localización de la membrana neovascular, la TFD constituye un posible tratamiento. Nuestra paciente respondió satisfactoriamente lo que se tradujo en una recuperación de la agudeza visual y en una regresión angiográfica de la lesión inicial. Por tanto, podemos decir que, la TFD puede ser un tratamiento eficaz en las membranas neovasculares secundarias a la presencia de estrías angioides (4) aunque, dada la diversidad de resultados en las distintas publicaciones (5), son necesarios estudios a largo plazo para esclarecer el verdadero potencial de la TFD en estos enfermos.

\section{BIBLIOGRAFÍA}

1. Blanco Rivera MC, Gómez Ulla De Irazazábal F, Ferrer Jaureguizar J, Abelenda Pose D. Angiografía verde indocianina en las estrías angioides. Arch Soc Esp Oftalmol 2001; 76: 297-302.

2. Piug J, García-Arumí J, Salvador F, Sararols L, Calatayud $M$, Alforja $S$. Neovascularización y hemorragias subretinianas asociadas a estrías angioides. Arch Soc Esp Oftalmol 2001; 76: 309-314.

3. Torrón Fernández-Blanco C, Pérez Oliván S, Ferrer Novella E, Ruiz-Moreno O, Marcuello Melendo B, Honrubia López FM. Terapia fotodinámica en membrana neovascular subretiniana no relacionada con degeneración macular asociada a la edad o miopía patológica. Arch Soc Esp Oftalmol 2003; 78: 471-476.

4. Menchini $U$, Virgili $G$, Introini $U$, Bandello $F$, AmbesiImpiombato $M$, Pece A, et al. Outcome of choroidal neovascularization in angioid streaks after photodymamic therapy. Retina 2004; 24: 763-771.

5. Arias L, Pujol O, Rubio M, Caminal J. Long-term results of photodynamic therapy for the treatment of choroidal neovascularization secondary to angioid streaks. Graefes Arch Clin Exp Ophthalmol 2006; 244: 753-757. 